\title{
Escort evolutionary game dynamics application on a distribution system with PV, BSS and EVs
}

\author{
Andres Ovalle, Ahmad Hably*, Seddik Bacha** \\ Univ. Grenoble Alpes, CNRS, Grenoble INP*, G2elab, GIPSA-lab, 38000 Grenoble, France. \\ * Institute of Engineering Univ. Grenoble Alpes \\ andres.ovalle-villamil@g2elab.grenoble-inp.fr
}

\begin{abstract}
This paper provides an optimal energy management application of a multipopulation approach based on the escort evolutionary game theory. A distribution system including photovoltaic $(\mathrm{PV})$ generation, a dedicated battery storage system (BSS) interacting with electrical vehicles (EVs) is studied. Different case studies with single-phase and three-phase EV chargers are presented and analysed. Results show the benefits of integrating a BSS regarding the undesired unbalancing effects and strong peaks of PV production.
\end{abstract}

Index Terms-Plug-In Hybrid Electric Vehicles, Smart charging, Vehicle-to-Grid, Peak shaving, Valley filling, Game theory, Escort evolutionary game theory.

\section{INTRODUCTION}

Electric Vehicles (EVs - fully electric and hybrid rechargeable vehicles) take advantage of large portable energy storage capacities to use clean and cheap electrical energy for transportation purposes. However, the integration of these vehicles to the grid may cause some technical impacts. Per the availability of resources (e.g. cloudy or windy days), micro-generators produce intermittent quantities of electricity, regardless of the hours of peak or low demand. On the other hand, EV owners expect to recharge their vehicles within residential electricity grids. Without any management method, many EVs plugging-in at the same time can easily congest the electricity infrastructures [1]-[3]. Furthermore, all these devices require interfaces (power electronics) to exchange energy with the grid. These interfaces have the inherent problem of polluting the system with harmonics that can affect other end users and infrastructural devices (transformers, industrial motors, etc.).

Without proper optimal management tools, these non-controlled perturbations are prone to destabilize the electrical system, provoking black-outs in the worst-case scenarios. To respond to these challenges, Energy Storage Systems (ESS) (e.g. batteries, or flywheels), along with adapted communication infrastructures and data analytics tools, appear as the cornerstone of the future smart electricity system. The benefits of energy storage in smart electricity grids can be understood by making an analogy to memory devices in informatics systems. ESS can reduce the impacts of intermittent micro-sources by storing generated energy for later use during peak hours. Keeping in mind this sense of purpose for ESS, if EVs are considered as a fleet instead of individual vehicles, they become part of the solution for smart electricity systems [4]. The approach of this present paper is to propose distributed optimization algorithms for the integral load management of electric vehicle fleets and their potential services to the electricity system. The proposed methodologies optimally manage EV fleets charge/discharge schedules by applying game theory, and evolutionary game theory techniques. These techniques are the application of population dynamical methods to game theory [5]. They can be employed to represent the evolution of the distribution of a population of a given species over multiple environments, depending on the resources these environments offer. They are suitable for load management problems. In [6], a game-theoretic model predictive control (MPC) approach for demand-side management that can be adapted to real-time data has been proposed. The authors of [7] have investigated a smart grid model with dynamic pricing for a population with distributed storage and generation capabilities. They have used a day-ahead optimization process in order to find a non-cooperative Nash equilibrium. A game-theoretic framework is established in [8] for modelling the strategic behaviour of buses that are connected to renewable energy resources.

In the approach proposed in this present paper, the energy consumed by an EV is represented by a population. Then, multiple populations can interact (multiple EVs interacting). These populations are distributed over multiple environments which in the model represents different moments of the day. Applying the mathematical representation of these techniques, decentralized optimization algorithms for EVs to provide multiple auxiliary services to the electricity grid can be proposed. These algorithms tackled the stochastic behaviour of variables like arrival and departure, initial and final state of charge, and incentives to EV owners. Without differentiation between single and three-phase EV chargers, the proposed methodologies seek to provide services like load flattening (shifting and shaving), load balancing among phases, reactive power supply, resource sharing among EVs, and interaction with renewable energy micro-sources.

The paper is organized as follows. Section II gives an overview of the proposed algorithm. Section III introduces and describes the system used in this paper. In section IV, the simulation results of the application of the algorithm are presented. Finally, section V gives some conclusions.

\section{OVERVIEW OF THE PROPOSED APPROACH}

The objective is to propose a solution to a global distributed problem, dealing with several local constrained variables. To accomplish this, a multi-population approach is proposed, as an application of the escort dynamics (ED) and the proposed escort functions of the recent work [9]. The ED describes the evolution of the distribution of a normalized population over $K$ possible pure strategies, according to the benefit provided by those strategies. This generalized escort evolutionary game dynamics is defined in continuous time by

$$
\dot{x}_{k}=\phi_{k}\left(x_{k}\right)\left(f_{k}(\mathbf{x})-\bar{f}_{\phi}(\mathbf{x})\right),
$$


where $\mathbf{x}=\left[x_{1}, x_{2}, \cdots, x_{k}, \cdots, x_{K}\right]^{\mathrm{T}}$ is the state vector of portions of the population following pure strategy $k, f_{k}(\mathbf{x})$ is the payoff function for strategy $k$, and $\bar{f}_{\phi}(\mathbf{x})$ is weighted with respect to the escort functions and the sum of them. The so-called escort function $\phi_{k}\left(x_{k}\right)$ can be understood as an incentive for the rate of growing. The following are some of the analogies proposed in this approach: Pure strategies are considered to be the phases at each time slot, while a population is a quantity to be split among several phases and slots of time (i.e. active and reactive powers). The whole number of populations depends on the number of connected electrical vehicles (EVs), while the size of each population and the number of pure strategies for each population depend on the owners' requirements. Each EV is locally in charge of two populations that represent respectively: Energy (Wh), and reactive power (var).

\section{A. Analogies for energy (Wh) populations}

In order to illustrate the analogies introduced in this paper, let us check Fig.1. In this diagram, three populations, with given number of individuals, are initially distributed among 12 environments identified by pairs $(k, m)$. Each environment has a given carrying capacity for individuals of the different populations, and each environment provides a certain payoff for those individuals. Among these three populations, the first one has a sedentary behavior, which means that its individuals are not willing to migrate to other environments. The individuals from the other two populations have nomad behaviors, i.e. they are prone to choose among environments in order to increase the payoff of their population. Each environment represents a pure strategy for each individual of the nomad populations, and each individual of these populations is able to choose among 12 pure strategies. The total number of individuals of the three populations does not change with time, i.e. the size of the total population is constant through the time. However, the nomad populations are able to convince individuals from the other populations to leave their current population and environment, and migrate. In this sense, the size of the two nomad populations, may increase with time at the expense of other populations' size decrease. Nonetheless, the size of the two nomad populations is limited by given reception capacities. Given this scenario, the size and distribution of the populations evolves with time. Fig. 1 shows initial and final sizes and distributions of the three populations according to the payoffs provided by the 12 environments.

This scenario summarizes an example of the proposed energy management application of this paper, for two EVs and four slots of time (four hours for instance). Each environment represents a phase $m=\{1,2,3\}$ at time $k=\{1,2,3,4\}$. The sedentary population represents the forecasted energy consumption at each phase of the transformer and at each slot of time. The carrying capacities of the environments represent the load limits of the transformer at each phase. Nomad populations represent the energy demands of each $\mathrm{EV}$ and the size of the population represents the amount of energy required to reach a desired state of charge of its battery. The idea of nomad populations attracting individuals from other populations represent the fact that connected EVs may consume energy from a phase at a given instant, and inject it at another phase and another instant. The reception capacity of a nomad population represents the battery capacity of the EV. Finally, the idea behind the evolution of the distribution of nomad populations reflects the fact that EVs may allocate consumption or injections of energy among phases and slots of time, depending on benefits they can get from, or services they can provide to the grid.

\section{B. Formal definition for the types of populations representing EVs variables}

An EV $i$ has to consume active power in order to fully charge its battery in a time window of length $K^{i}$. This length is defined or estimated by the owner who can be as restrictive as she/he requires (taking into account the charger's limits). In the case of three-phase chargers, the consumption can be distributed among slots of time and phases. Thus, a phase at a given time slot is represented by one of multiple pure strategies. The following set of constraints is obtained:

$$
\begin{aligned}
& \sum_{k=1}^{K^{i}} \sum_{m=1}^{3} x_{k, m}^{i}=s o c_{d}^{i}-\operatorname{soc}_{0}^{i} \\
& x_{k, m}^{i} \leq \overline{s o c}^{i}-\left(\operatorname{soc}_{0}^{i}+\sum_{\omega=1}^{\Omega} \sum_{m=1}^{3} x_{\omega, m}^{i}-x_{k, m}^{i}\right) \\
& x_{k, m}^{i} \geq \underline{s o c^{i}}-\left(\operatorname{soc}_{0}^{i}+\sum_{\omega=1}^{\Omega} \sum_{m=1}^{3} x_{\omega, m}^{i}-x_{k, m}^{i}\right) \\
& \forall \Omega=\left\{1,2, \cdots, K^{i}\right\}, \forall k=\{1,2, \cdots, \Omega\}, \forall m=\{1,2,3\} \\
& -t_{k}^{i} \bar{p}^{i} \leq x_{k, m}^{i} \leq t_{k}^{i} \bar{p}^{i}, \\
& x_{k, m}^{i} \leq t_{k}^{i}\left(\bar{L}-\pi_{k, m}\right)+x_{k, m}^{i} \\
& \forall k=\left\{1,2, \cdots, K^{i}\right\}, \forall m=\{1,2,3\}
\end{aligned}
$$

where $s o c_{d}^{i}$ is the desired state of charge (Wh) at the end of the time window defined by the owner, $s o c_{0}^{i}$ is the initial state of charge (Wh), $t_{k}^{i}$ is the length of the step of time $k$ in hours or fractions of hour, $\bar{p}^{i}$ is the nominal power of the charger per phase, and $\bar{L}$ is the limit load of the transformer per phase. Constraints (2b) and (2c) represent the accumulated state of charge at time $\tau$ which cannot go below $\underline{s o c}^{i}$ or above $\overline{s o c}^{i}$. Constraint (2d) represents the limits of energy consumption/injection given the nominal limits of the charger and the length of the step of time $k$. Constraint (2e) represents the limits of energy consumption given the nominal limits of the transformer and the length of the step of time $k$.

\section{Payoff function definition}

In this approach, payoff functions take into account both the objectives of the utility grid manager and the EV owners. Two factors are defined. The first factor is a commitment factor $\mu^{i}$ controlled by EV owners. It gives them some choice regarding their commitment to the gird on one hand and the ability of utility grid manager on the other hand. This factor is given as follows:

$$
\underline{\mu} \leq \mu^{i} \leq 1
$$

where $0 \leq \underline{\mu} \leq 1$ is the minimum allowed level, and 1 is the maximum level of commitment. The second factor is a smoothing factor $\eta$. This factor is controlled by the utility grid manager in order to manage the variations of total active and reactive power profiles over time. It is given as follows:

$$
0 \leq \eta \leq 1
$$

In this approach, monotonically decreasing payoff functions are proposed and the candidate functions are quadratic, strictly concave on all the controllable parameters. The level of commitment and the smoothing parameter define the zero crossing of these candidate 


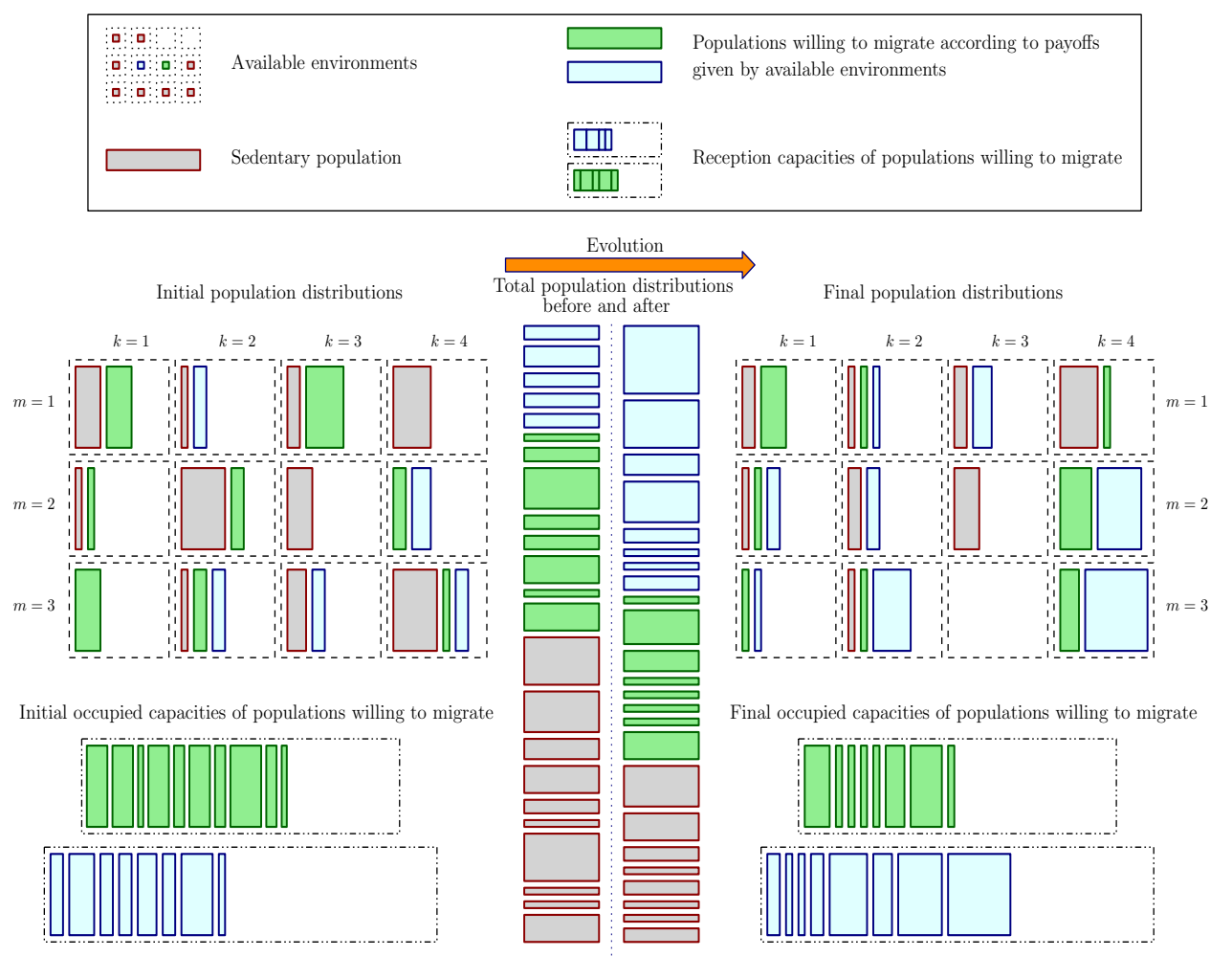

Fig. 1. Illustrative example of the multi-population approach proposed for the distributed energy management.

functions. For Energy pure strategies the proposed payoff functions are,

$$
\begin{aligned}
& f_{k, m}^{i}\left(x_{k, m}^{i}\right)=-\left(1-\mu^{i}\right)\left(\frac{x_{k, m}^{i}-x_{k, m}^{i *}}{t_{k}^{i}}\right) \\
& -\mu^{i}\left[\eta \pi_{k, m}+(1-\eta)\left(2 \pi_{k, m}-\pi_{k-1, m}-\pi_{k+1, m}\right)\right],
\end{aligned}
$$

where $x_{k, m}^{i *}$ is the owner's preferred reference for the charging rate (i.e. the reference for power consumption of the charger at time $k$ and phase $m$ ). For single-phase chargers, an additional parameter $v$, such that $0 \leq v \leq 1$, is included in order to balance between single-phase grid's interests $(v=0)$ and three-phase grid's interests $(v=1)$.

\section{The role of aggregator}

An aggregator is in charge of managing the interaction among EVs following a Best Reply approach [10]. It receives optimal profiles from EVs, then it compiles the information adding the EV profiles to the forecasted active and reactive power profiles, and redistributes them to EVs again, making the new information available to EVs under the transformer in a certain order. Finally, given historic data [11], the aggregator provides an updated 24-hour forecast of the loading of each phase of the MV-LV transformer.

\section{THE STUDIED SYSTEM}

The multi-population escort evolutionary game theory approach of this paper is tested on a system including photovoltaic generation, a dedicated battery storage system interacting with EVs. Real historic data has been used. It has been collected from a distribution transformer of the SOREA utility grid company, in the region of Savoie, France. The purpose of this test, is to check how
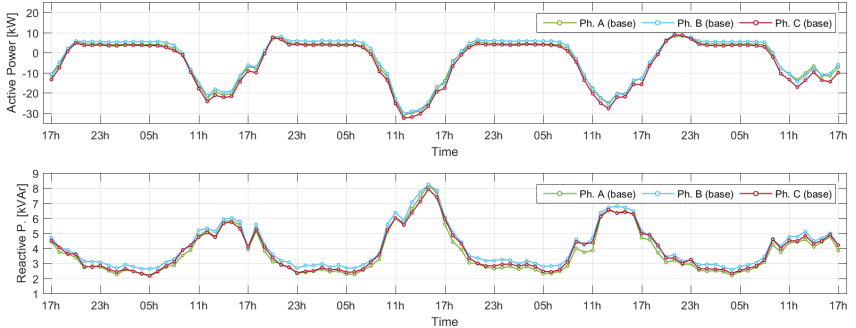

Fig. 2. Active and reactive power profiles for the four day test scenario with photovoltaic (PV) generation, and a dedicated battery storage system (BSS). Forecasts based on real historic data measured from a transformer for "sports facilities', from the SOREA utility grid company in the region of Savoie, France.

the proposed approach behaves when there is a high penetration of renewable energy, and an additional dedicated storage system is able to interact with the connected EVs. Besides it is also useful to check a scenario where the load among phases is well balanced, as it is evidenced by the historic data measurements. Furthermore, under this transformer, three sets of photovoltaic modules, with peak capacities of $60 \mathrm{kWp}, 60 \mathrm{kWp}$, and $80 \mathrm{kWp}$, are installed. On the other hand, a group of batteries with an energy storage capacity of $36 \mathrm{kWh}$ at a nominal power of $18 \mathrm{~kW}$, is currently being installed. The load under this transformer, corresponds completely to sports facilities. A summary of the conditions of this case study is given on Table I.

For this system, four spring days of forecasted active and reactive powers per phase in hourly steps, are shown on Fig. 2. It can be noticed that the consumption/production, and reactive power of the 
TABLE I

DESCRIPTIVE SUMMARY AND ASSUMPTIONS OF THE CONSIDERED REALISTIC SCENARIO WITH PV GENERATION, AND DEDICATED BSS.

\begin{tabular}{|c|c|}
\hline Item & Description \\
\hline Dedicated BSS & $\begin{array}{l}36 \mathrm{kWh} \text {, nomimal rate of charge power } \\
18 \mathrm{~kW}\end{array}$ \\
\hline PV arrays & 2 of $60 \mathrm{kWp}$, and one of $80 \mathrm{kWp}$ \\
\hline Chargers & $\begin{array}{l}3.3 \mathrm{~kW} / \text { phase with probability of } 80 \% \\
7.5 \mathrm{~kW} / \text { phase with probability of } 20 \%\end{array}$ \\
\hline Batteries & $\begin{array}{l}8.8 \mathrm{kWh} \text { with probability of } 30 \% \\
20 \mathrm{kWh} \text { with probability of } 70 \%\end{array}$ \\
\hline Constraints on batteries & $\begin{array}{l}\text { Between } 25 \% \text { and } 90 \% \text { for } 8.8 \mathrm{kWh} \\
\text { Between } 30 \% \text { and } 85 \% \text { for } 20 \mathrm{kWh}\end{array}$ \\
\hline Single or 3-phase charger & $\begin{array}{l}\text { Probability of } 50 \% \text { for single-phase } \\
\text { and } 50 \% \text { for three-phase charger } \\
7.5 \mathrm{~kW} / \text { phase with probability of } 20 \%\end{array}$ \\
\hline Connection phase & Probability of $33.33 \%$ for each phase \\
\hline Time period & 4 days (96 hour steps) \\
\hline Highest rate of arrivals & $7 \mathrm{EVs} / \mathrm{h}$ at $05 \mathrm{~h}$ \\
\hline Lowest rate of arrivals & $0.5 \mathrm{EV} / \mathrm{h}$ at $04 \mathrm{~h}$ next day \\
\hline Peak of connected EVs & $\begin{array}{l}\text { Between } 24 \text { and } 32 \text { EVs around } 09 \mathrm{~h} \text { each } \\
\text { day. }\end{array}$ \\
\hline Trade-off values $\mu^{i}$ & Common and fixed for all EVs to $\mu^{i}=0.9$. \\
\hline Utility grid manager & Service parameter fixed at $\eta=0.7$ \\
\hline Utility grid manager & Service trade-off factor $v=0$ \\
\hline Distribution system info. & $\begin{array}{l}\text { Data from SOREA utility grid company, } \\
\text { Savoie, France }\end{array}$ \\
\hline Scenarios & $\begin{array}{l}\text { 1. Single-phase chargers only } \\
\text { 2. Both single and three-phase chargers }\end{array}$ \\
\hline
\end{tabular}

a)
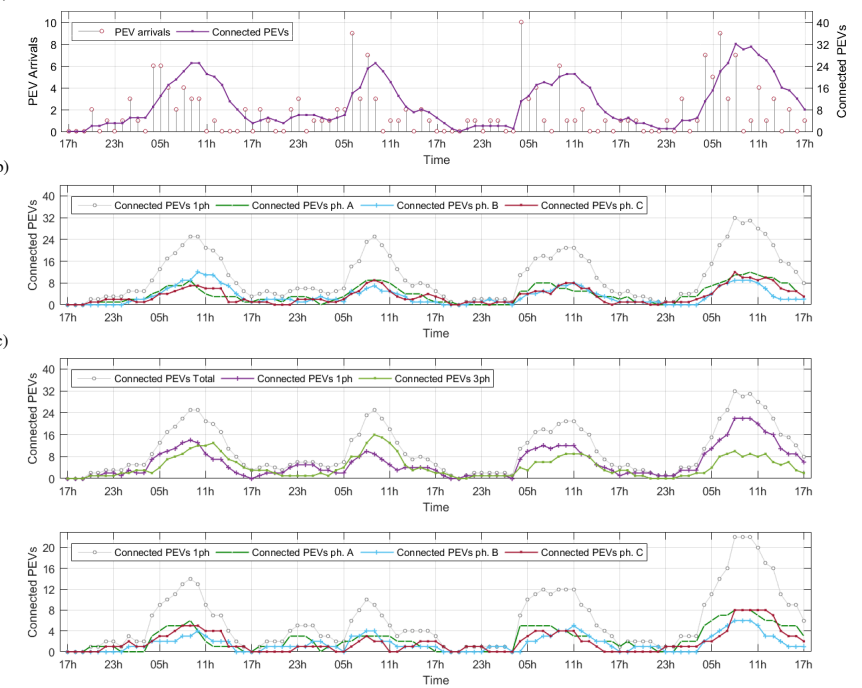

Fig. 3. (a) Profiles for the total amount of connected vehicles in the single-phase chargers only scenario. The number of arriving EVs each hour, for the four days test scenario, is shown as well. (b) The total amount, and the amounts of vehicles connected per phase, for the single-phase chargers only scenario. (c) For the scenario with both types of chargers: The amount of vehicles connected to threephase and single-phase chargers, and the amount of EVs connected to single-phase chargers per phase.

system are well balanced among the three phases. Moreover, it can noticed that consumption is usually much less than the PV production. As a consequence an important amount of energy is injected to the grid through the transformer each day.

On the other hand, let us consider the profile of arrivals and departures of EVs, shown on Fig. 3(a). This profile is obtained using
Profiles for single-phase chargers only $(v=0)$, and PV generation
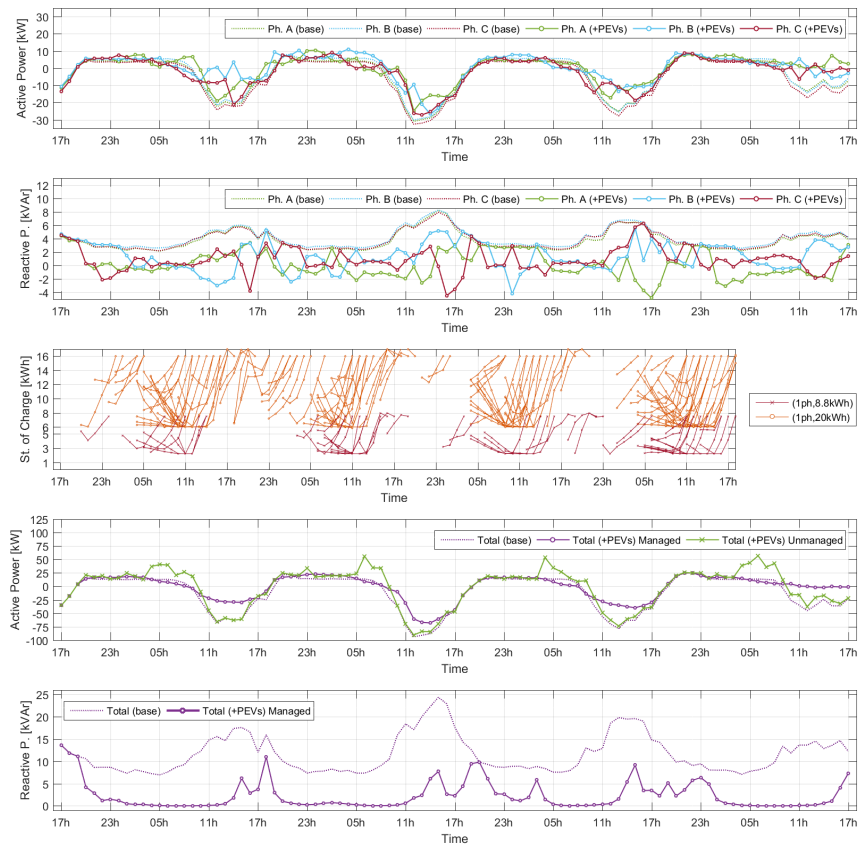

Fig. 4. Case study with single-phase chargers $(v=0)$, several days and a realistic scenario with photovoltaic (PV) generation. (a) Forecasted and final active power profiles, for each phase, obtained with the proposed approach. (b) Forecasted and final reactive power profiles, for each phase, obtained with the proposed approach. (c) State of charge profiles for each EV, obtained with the proposed approach. (d) Forecasted and final total active power profiles, obtained with the proposed approach. Comparison with unmanaged case. (e) Forecasted and final total reactive power profiles, obtained with the proposed approach.

a classical Poisson model with variable rate of arrivals (changing with time) and variable connection times. The highest rate of arrivals is $7 \mathrm{EVs} / \mathrm{h}$ at $05 \mathrm{~h}$ and it decays up to $0.5 \mathrm{EVs} / \mathrm{h}$ at $04 \mathrm{~h}$ the next day. Moreover two, likely to occur, scenarios are considered: when there is only presence of single phase chargers, and when there is presence of both three and single-phase chargers in equal proportions in the grid. For the first of these two scenarios, Fig. 3(b) shows the profile of total connected EVs through the studied days, as well as the distributions of those EVs on single-phase chargers connected over the three phases. For the second scenario, Fig. 3(c) shows the same profile of total connected EVs through the study days, and this time, the distributions of those EVs among single-phase and three-phase chargers. For the corresponding portion of EVs connected to singlephase chargers, this figure shows the distribution over the threephases as well.

\section{RESUlts}

Fig. 4 shows the behavior of the proposed ED approach over the interesting variables of the system, when only single phase chargers are considered and $v=0$. The applied arrivals and departures profiles, are those previously mentioned on Figs. 3(a) and 3(b). As it can be expected, the presence of single-phase chargers only, has a negative effect on the balance of load among phases, as it can be observed for the active and reactive power profiles on Figs. 4(a), 4(b). 
Profiles for single-phase chargers only $(v=0), \mathbf{P V}$ generation, and dedicated batteries
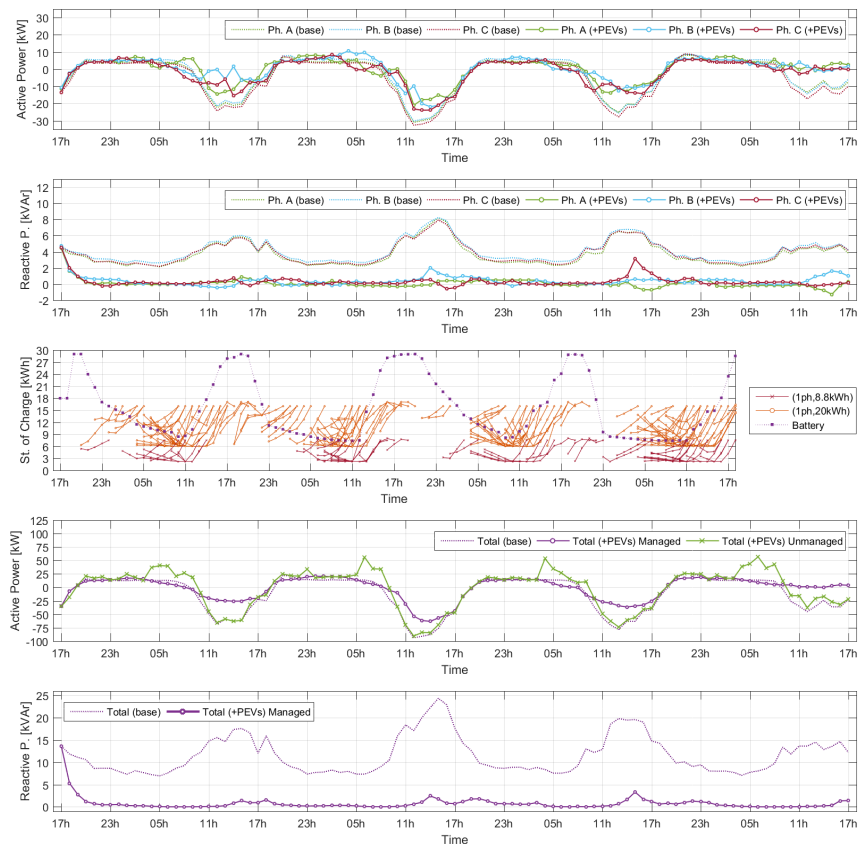

Fig. 5. Case study with single-phase chargers $(v=0)$, several days and a realistic scenario with photovoltaic (PV) generation, and a dedicated battery storage system (BSS). (a) Forecasted and final active power profiles, for each phase, obtained with the proposed approach. (b) Forecasted and final reactive power profiles, for each phase, obtained with the proposed approach. (c) State of charge profiles for each EV, obtained with the proposed approach. (d) Forecasted and final total active power profiles, obtained with the proposed approach. Comparison with unmanaged case. (e) Forecasted and final total reactive power profiles, obtained with the proposed approach.

Similar profiles can be observed in Figs. 5(a), 5(b), where, besides the same presence of single-phase chargers, the BSS interacts as well with EVs. The BSS has a capacity of $36 \mathrm{kWh}$, with states of charge limited to cycles between $20 \%$ and $80 \%$ (a useful capacity of around $22 \mathrm{kWh})$, and a nominal power of $18 \mathrm{~kW}(6 \mathrm{~kW} /$ phase $)$. In the proposed approach, the BSS interacts with EVs, as another $\mathrm{EV}$, through the aggregator. Its desired state of charge is always $18 \mathrm{kWh}(50 \%)$, and its corresponding "populations" of active and reactive power are always distributed among 36 environments, corresponding to the 3 phases during a connection time horizon of 12 hour steps, $m=\{1,2,3\}$, and $k=\{1,2, \cdots, 12\}$. Off course this time horizon can be longer or shorter depending on the utility grid manager criteria.

Comparing the results for only single-phase chargers, with those when the BSS is included, it can observed that the BSS strongly mitigates the undesirable unbalancing effects of single-phase chargers, specially in terms of reactive power. Concerning, the states of charge in Fig. 4(c), without battery, and $v=0$, single-phase chargers are always focused on the smoothing/flattening objectives for total load profiles, so chargers always try to shift their consumption to those hours where PV produces the deepest values of total active power. With the BSS, the behavior is similar, as it can be observed in Fig. 5(c). The presence of the BSS allows to balance the effects of singlephase chargers in different phases, by modulating the consumption
Profiles for three-phase, single-phase chargers $(v=0)$, and PV generation
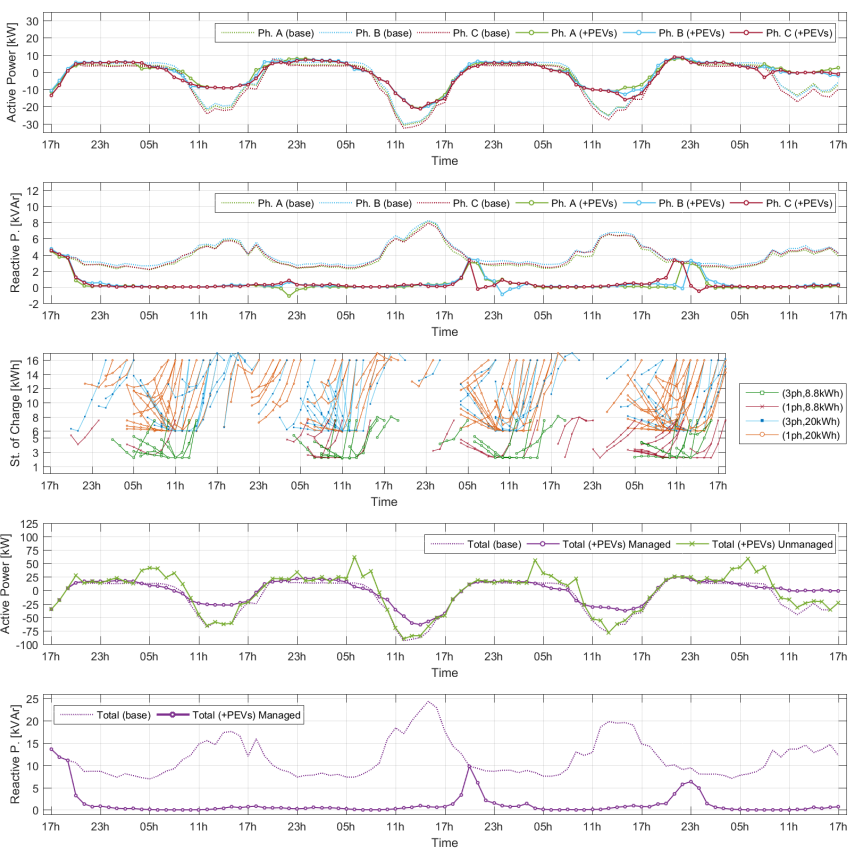

Fig. 6. Case study with both three-phase and single-phase chargers $(v=0)$, several days and a realistic scenario with photovoltaic (PV) generation. (a) Forecasted and final active power profiles, for each phase, obtained with the proposed approach. (b) Forecasted and final reactive power profiles, for each phase, obtained with the proposed approach. (c) State of charge profiles for each EV, obtained with the proposed approach. (d) Forecasted and final total active power profiles, obtained with the proposed approach. Comparison with unmanaged case. (e) Forecasted and final total reactive power profiles, obtained with the proposed approach.

of its phases in consequence. The state of charge cycles of the BSS evidence high consumption rates during hours of deep values of total active power, which reflect as well, the smoothing/flattening objectives. On the other hand, given that single-phase chargers consume as much as they can during the hours of high power production (aiming to accomplish the smoothing/flattening objectives), there is a lack of available reactive power from EVs and they are not able to supply the demand of the grid. This can be observed in Fig. 4(e), especially around $17 \mathrm{~h}$ on each day. On the contrary, when the BSS is available, it also consumes as much as it can during the peaks of PV production, but around $17 \mathrm{~h}$ each day, it has already reached states of charge close to the upper limit of $80 \%$ (29kWh). Because of this, around $17 \mathrm{~h}$ each day, its consumption is limited in order to avoid surpassing this limit, and as a consequence, it has available reactive power in order to supply the grid demand. The difference can be appreciated by checking the total reactive power profile in Fig. 5(e).

When both single-phase and three-phase chargers are considered, three-phase chargers are enough to mitigate the balancing issues caused by single-phase chargers. This can be observed on Figs. 6(a) and 6(b), for active and reactive power profiles of individual phases, when the arrivals and departures profile in Fig. 3(c) is considered. However, thee-phase chargers are also intended to consume as much as possible during strong PV production, so their available reactive power is also reduced. The effects are not as string as for singlephase chargers, but still, around $17 \mathrm{~h}$, the reactive power demand of 
Profiles for three-phase, single-phase chargers $(v=0)$, PV generation, and dedicated batteries
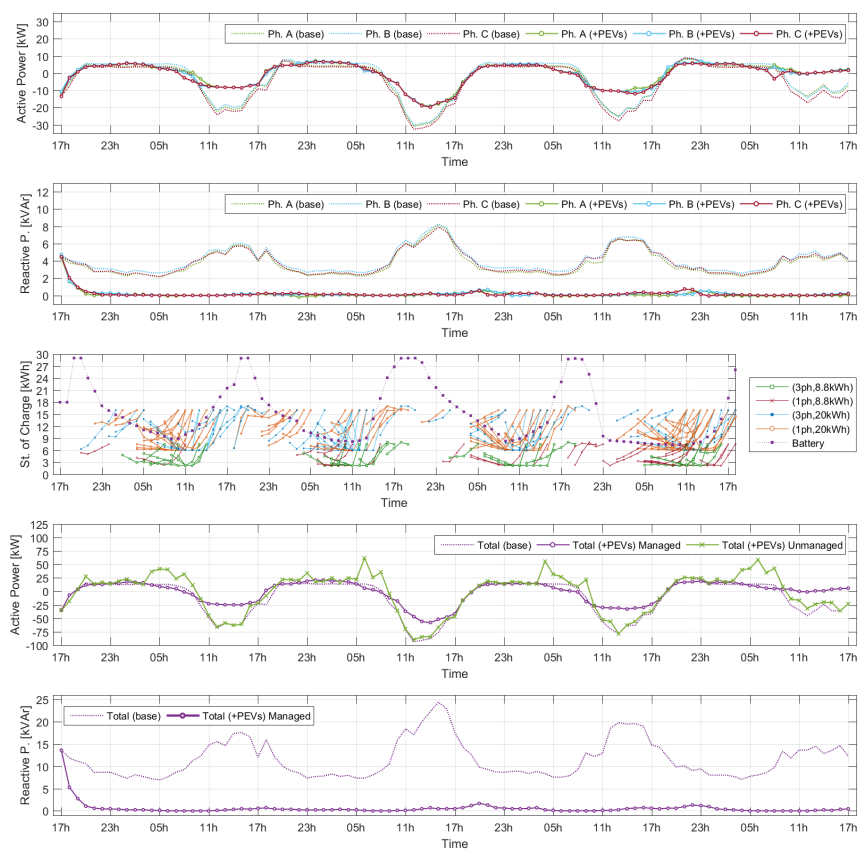

Fig. 7. Case study with both three-phase and single-phase chargers $(v=0)$, several days and a realistic scenario with photovoltaic (PV) generation, and a dedicated battery storage system (BSS). (a) Forecasted and final active power profiles, for each phase, obtained with the proposed approach. (b) Forecasted and final reactive power profiles, for each phase, obtained with the proposed approach. (c) State of charge profiles for each EV, obtained with the proposed approach. (d) Forecasted and final total active power profiles, obtained with the proposed approach. Comparison with unmanaged case. (e) Forecasted and final total reactive power profiles, obtained with the proposed approach.

the grid cannot be completely supplied by the EVs. On the contrary, comparing Figs. 6(e) and 7(e), when the BSS is introduced, the supply of reactive power is almost completely assured, in collaboration with EVs.

Comparing the total active power profiles of the two considered scenarios, and the inclusion of a dedicated energy storage system on both of them, on Figs. 4(d), 5(d), 6(d), and 7(d), it is possible to observe that the presence of PV generation forces EVs to shift their consumption mostly to peak hours of production. This motivates the use of clean energy sources and improves the technical behavior of the grid under the existence of this type of energy sources. Moreover, the existence of a dedicated energy storage system, of a relatively small size, could complement the ancillary services that EVs are able to provide with the proper coordination provided by the proposed approach. Besides, the diversity on types of connected chargers (three-phase and single-phase) is beneficial for the grid: it is able to improve its power quality, while it introduces the proper use of renewable energy sources, it provides the charging service to the EV owners, and it reduces infrastructure maintenance costs.

\section{CONCLUSION}

In this paper, a dedicated battery storage system (BSS) system is included in the loop of synergies induced by a multi-population approach for distributed management of EV load. In this scenario, multiple photovoltaic modules are connected to a distribution transformer feeding a sports facility. Moreover, according to the on-site measurements, the system is well balanced before the inclusion of EV chargers. The dedicated BSS is proposed to act as another EV interacting with the others through the aggregator. It is observed that the inclusion of the BSS strongly mitigates the undesired unbalancing effects produced when only single-phase chargers are considered, and single-phase grid's interest are privileged over threephase grid's interests. When there is presence of both three-phase and single-phase chargers, three-phase chargers are enough to mitigate the unbalancing effects of single-phase chargers. However, the strong peaks of PV production force all EVs to consume. Consequently they are not able to supply the reactive power demand during those hours. In that scenario, it was observed that the BSS reinforces the service provided by EVs given its larger nominal power. It is important to notice again that, under the proposed approach, the peaks of PV production force EVs to shift their consumption to these peak hours of production. This correlation motivates the installation of renewable energy sources together with EV chargers, aiming to use clean energy sources while their intermittency is mitigated by EVs and dedicated BSSs.

\section{REFERENCES}

[1] H. Turker, S. Bacha, D. Chatroux, and A. Hably, "Low-voltage transformer loss-of-life assessments for a high penetration of plug-in hybrid electric vehicles (phevs)," IEEE Trans. Power Del., vol. 27, no. 3, pp. 1323-1331, July 2012.

[2] B.-G. Kim, S. Ren, M. van der Schaar, and J.-W. Lee, "Bidirectional energy trading and residential load scheduling with electric vehicles in the smart grid," IEEE Journal Selected Areas in Comm., vol. 31, no. 7, pp. 1219-1234, July 2013.

[3] K. Kumar, B. Sivaneasan, P. So, and D. Wang, "Methodology for optimizing the number of electric vehicles deployed under a smart grid," in IECON 2013 - 39th Annual Conf.IEEE Ind. Elec. Soc., Nov 2013, pp. $4647-4652$.

[4] R. Couillet, S. Perlaza, H. Tembine, and M. Debbah, "Electrical vehicles in the smart grid: A mean field game analysis," IEEE Journal Selected Areas in Comm., vol. 30, no. 6, pp. 1086-1096, July 2012.

[5] J. Hofbauer and K. Sigmund, Evolutionary Games and Population Dynamics. Cambridge University Press, 1998.

[6] E. R. Stephens, D. B. Smith, and A. Mahanti, "Game theoretic model predictive control for distributed energy demand-side management," IEEE Transactions on Smart Grid, vol. 6, no. 3, pp. 1394-1402, 2015.

[7] I. Atzeni, L. G. Ordóñez, G. Scutari, D. P. Palomar, and J. R. Fonollosa, "Noncooperative and cooperative optimization of distributed energy generation and storage in the demand-side of the smart grid." IEEE Trans. Signal Processing, vol. 61, no. 10, pp. 2454-2472, 2013.

[8] Q. Zhu, J. Zhang, P. W. Sauer, A. Domínguez-García, and T. Başar, "A game-theoretic framework for control of distributed renewable-based energy resources in smart grids," in American Control Conference (ACC), 2012. IEEE, 2012, pp. 3623-3628.

[9] A. Ovalle, A. Hably, and S. Bacha, "Alternative defnitions of escort functions in the escort evolutionary dynamics with stability proof," Automatica, vol. submitted, 2015.

[10] J. Hofbauer and K. Sigmund, "The theory of evolution and dynamical systems." Cambridge University Press, 1988.

[11] K. Basu, L. Hawarah, N. Arghira, H. Joumaa, and S. Ploix, "A prediction system for home appliance usage," Energy and Buildings, vol. 67, no. 0, pp. $668-679,2013$. 